\title{
Express Automatic Sorting Machine
}

\section{Tian-Yang LIANG, Fei ZHONG, Xin YUAN, Di YAO, Nian YUE, Zhong-Wei CHENG}

Hubei University of Technology, Wuhan, Hubei 430000

$577957271 @ q q . c o m$

Keywords: Express automatic sorting, Visual system, Tilt sorting mechanism.

\begin{abstract}
Express automatic sorting is one of the main problems in the domestic logistics and transportation section. At present only a few large domestic and foreign logistics companies possess automated logistics sorting lines. The sorting equipment is complex in structure, large in size, high in cost and energy consumption. This paper presents a FedEx automatic sorting equipment for conveying belt transmission by visual identification, information analysis, and matching the data terminal and the courier company. This essay will introduce a kind of gradient express sorting mechanism, through the implementation of efficient and accurate sorting to express, can effectively solve the problem of sorting efficiency, equipment cost and avoid the sort of violence effectively. This scheme provides a compact express automatic sorting device, which provides a certain way of thinking for the automatic sorting of express delivery.
\end{abstract}

\section{Introduction}

With the rapid development of E-commerce as well as express logistics, each courier company faced with the number of courier sorting, heavy workload and other issues. At the same time people's concerns about the E-commerce are constantly increasing. After preliminary market research, found that express in transport part, from business acceptance to express delivery to the hands of consumers, will eventually need a total ten sections through information collection, sorting, packaging of goods goods collection and distribution.

According to the survey Ewhich found that there are part of the automatic sorting lines exist in domestic and foreign express delivery company's core distribution center, At present, only in the domestic SF and postal express transit center to express relatively large automatic sorting line mature, the rest of the courier companies are using way of "labor + transporting belt" to sort express. However, there is no apparatus like transporting belt available in remote areas, lack of financial support is the fundamental reason accounting for this phenomenon. SF is an imitation of FedEx and UPS. In sum, equipment investment is large, so many enterprises are lack of processing capacity at home and abroad. Now the strongest foreign enterprises are Dema Taco, Van Trend, Switzerland. Domestic sorting equipment mostly belong to copy the sorting equipment of these companies and the sorting quality is not stable. This is a big limitation of the development of the domestic express, as well as the reason for the low efficiency.

The domestic courier has three main categories: the first category of corrugated packaging, with regular shape, second is cuboid; clothing and other soft objects in the packaging, usually regarded as a cuboid; third, a kind of surface, which is usually the thickness of the envelope. Given that some of the courier package size are relatively large, and their quantity is small, thus they are not taking into account when sorting. It goes the same for the small size of the courier, the bar code may be glued to the two face, which cannot be identified by visual identification system, as mentioned above, and this design is designed to solve the regular size of the courier sorting problem. The express automatic sorting machine is simple and can effectively achieve the high quality of the fast sorting, reduce the cost of equipment is the goal of this design effectively.

Up to now, China's express delivery is still mainly rely on the courier staff to complete the entire transport process of all the work. This essay puts forward a new idea of automatic sorting equipment, which can overcome above problems effectively, complete of the delivery of the sorting 
work effectively, and avoid the problem of violent loading and unloading.

\section{Express Sorting Machine Research Ideas}

Through the understanding of the most current express type and size. Knowing that the discrepancy of each express is huge, and posted details of a single is not fixed. The direction of Express flow across the country. Visual recognition system, data sharing and sorting mechanism for the computer terminal to provide ideas for solving these problems. Using the visual system to identify the information of express delivery, with the computer terminal data, through the sorting mechanism to achieve the sorting of the courier, thus replacing the traditional manual sorting method.

Study objectives: to design the reasonable appearance structure of express sorting machine , transport mechanism, sorting mechanism, automatic sorting function to express the conventional Express (size length $*$ width $*$ height in the range of $200 \mathrm{~mm} * 200 \mathrm{~mm} * 200 \mathrm{~mm}$ to $800 \mathrm{~mm} *$ $800 \mathrm{~mm} * 800 \mathrm{~mm}$ in the sorting and delivery of information). Improving the efficiency of sorting and avoid violent loading and unloading. To design efficient and portable express sorting machine.

During the process of sorting express, because of a large quantity of express, which requires a fast sorting speed, so the equipment is installed with automatic correction device. After the courier box placed on the express sorting machine, express bill may be distributed at a random direction, therefore the express's two-dimensional code visual recognition system must be able to identify six directions express two-dimensional code, namely containing at least five vision cameras. Few sorting are required during the process that expresses are taken out of the warehouse and delivered to consumers. The addresses which will be sorted are also of great number. This equipment adopts a new sorting device, using the slant rendering expresses slide downward by their own weight, this method not only reduces the size of the sorting machine, also reduces the energy consumption of the equipment. According to the demanding usage, a different number of opening gates are arranged on the inclined plane, and using the cylinder to control the on -off of the gate.

During the process of express sorting, there may be some expresses cannot identify, if such were the case, putting into the storage area cannot identify the preset (this design is not recognized by the courier slid to the bottom of the slope sorting mechanism).

\section{The Design of the Fast Express Sorting Machine}

\section{The Overall Scheme of the Sorting Machine}

The device is intended to be used as shown in Figure 1, the overall structure of the sorting machine is easily adjustable to different heights of express vehicles. The whole device is compact, simple and portable, low energy consumption and light weight.

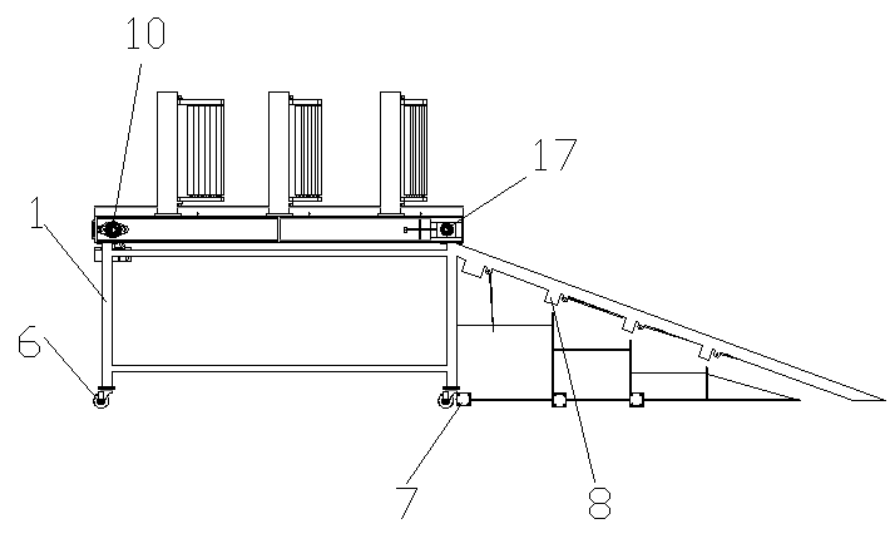

Figure 1 


\section{The Working Principle of Express Sorting Machine and Content}

Through the analysis of the express sorting, bar code recognition mechanism in the visual recognition system, the express sorting machine are comprised of a frame, angle steel, channel steel, drive motor, and cycloid reducer, cycloid reducer through the coupling shaft and the belt drive roller is connected to power transmission belt transparent. The angles are fixed on the brush bracket (three groups), the rolling brush and the rolling brush bracket through a hinge connection. The courier in the sorting near the rolling brush right drive motor side brush holder, the brush bracket in the middle of the three roll rolling wall and transparent belt opposite install a set of visual system on the brush rack, left, down, to express a single full range of recognition.

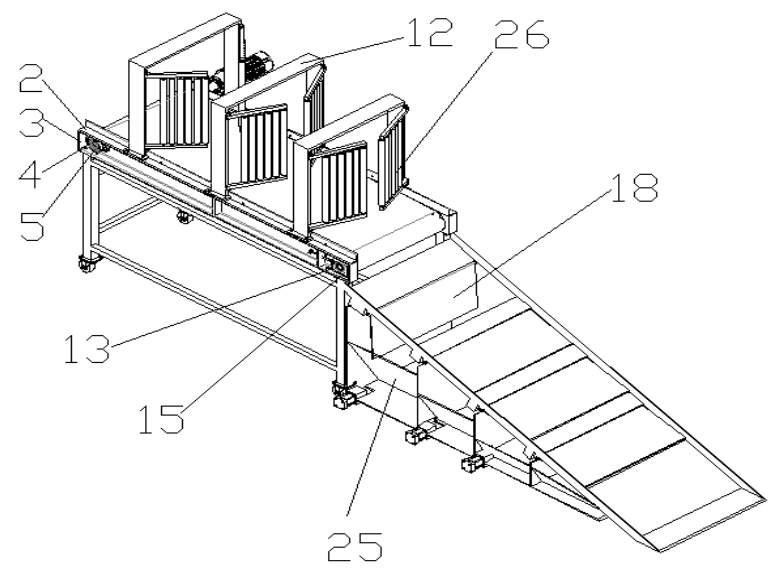

Figure 2

In the front of the channel front, both sides were casting diamond fixed deep groove ball bearings, belt drive roller is installed in the inner deep groove ball bearing. In the end of steel belt, roller bearings mounted in the bearing sleeve through the combination of the bearing sleeve and the tension block is fixed to the bearing housing assembly, track fixed on the end of the channel. The screw through hole on the fixed rail bearing sleeve is connected with the tension adjusting block, tension mechanism is realized by screwing the screw. A universal wheel for the lower part of the machine frame. At the end of the drive motor, the third group of rolling brush holder is controlled by the microcomputer, and the express box is put on the sorting mechanism in order. Conveying belt end connection inclined type sorting mechanism. Tilt type sorting mechanism comprises an inclined sorting plate, a sorting plate, a sorting and rocking plate cylinder. The sorting and shaking plate cylinder is fixed below the inclined sorting plate of the sorting mechanism, and is controlled by the sorting and shaking plate motor. The first sorting can be realized through the sorting plate. The sorting plate is arranged under the push plate roll sorting and sorting push plate cylinder, the push plate cylinder by sorting sorting push plate of motor control, in the chute of the frame under the constraints of the linear reciprocating motion of the second sorting to express.

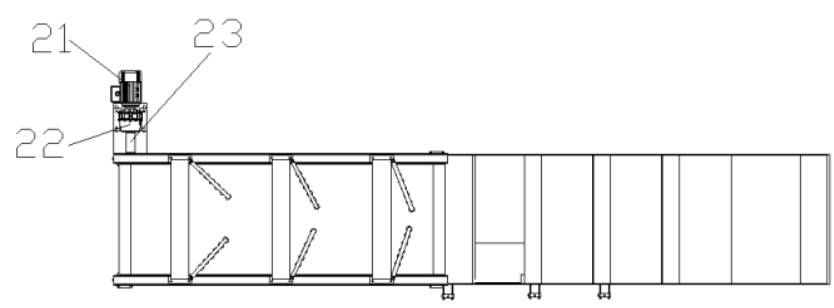

Figure 3 
Express box will be dropped at the specified location, the final sorting will be initiated by the force exerted by the push plate. Non-identified expressed will be dropped to the bottom and ready for the next sorting.when Sorting is completed according to the requirements, the expresses will be packed together and be transported or delivered.

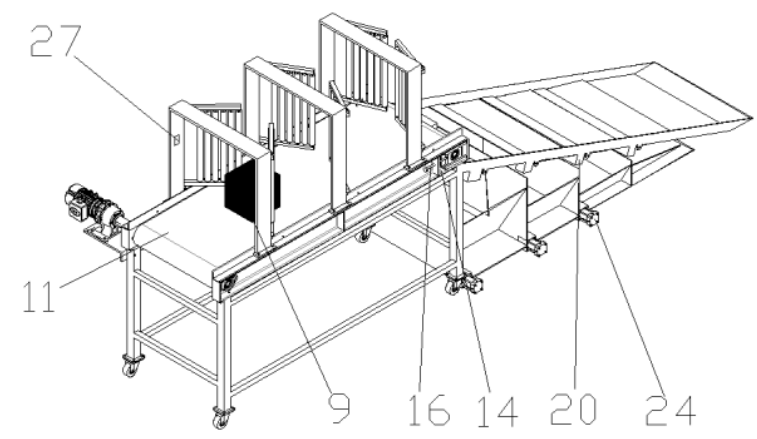

Figure 4

\section{Express Sorting Machine Belt Tension Device}

The belt tension device is shown in Figure 5. The structure is simple and portable, easy to control, low cost and easy to implement.

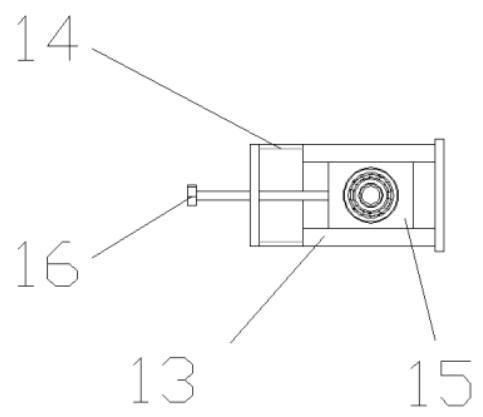

Figure 5. belt tension device

\section{The Efficiency Calculation of the Express Sorting Machine}

Express sorting machine opts for three-phase asynchronous motor, model for the YSJ6324-IP55-0.18KW, speed $\mathrm{n}=1400 \mathrm{r} / \mathrm{min}$. Cycloid reducer adopts $\mathrm{B}$ series cycloid reducer horizontal installation, model BWD-10-18-0.55, with the use of motor, gear ratio $\mathrm{i}_{12}=17$, transmission efficiency $\eta \geq 90 \%$. The roller conveyor belt on rigid coupling and reducer connected power $(\eta \geq 90 \%)$, drum diameter $\mathrm{D}=120 \mathrm{~mm}$. Conveyor belt length $\mathrm{L}=1650 \mathrm{~mm}$.

The sorting efficiency is 30 pieces per minute. The speed of the transmission belt

$$
\mathrm{V} 1=\mathrm{V} 2=(\mathrm{n} / 60 * \pi \mathrm{D}) / \mathrm{i} 12=0.5 \mathrm{~m} / \mathrm{s}
$$

The frame is simply supported beam, the material is Q235, 1750mm long, distance $710 \mathrm{~mm}$, through the beam bending check of normal stress, bending stress $\sigma$ and shear Forcetmeet the strength requirements.Because this design for the regular express sorting, small size, light weight, frame strength, the overall structure to meet the needs of the use, not to do a list.

\section{Conclusion}

Domestic E-commerce are developing rapidly, the efficiency and quality of the logistics exert an impact on the E-commerce, E-commerce holds a mounting higher requirement to the logistics. Express automatic sorting machine provides a promising platform for the intelligence tasks of express sorting. At present, only few courier companies in the core of the sorting area possess 
automatic sorting line, equipment costs are expensive, stability needs to be improved. This essay presents a low cost, simple and compact structure, low energy consumption of express sorting equipment. It provides some ideas for the logistics transportation and sorting.

Through the research of this project, we can not only improve the efficiency of express sorting, avoid the brutal loading and unloading, but also put forward a new type of sorting mechanism. The complexity of the sorting line is reduced, and the equipment cost is reduced. At the same time, it has important significance to improve the degree of automatic operation of express delivery companies and obtain higher economic benefits and social benefits.

\section{Reference}

1. Wu Like, CEN Quefu. Analysis of multifunctional vehicle and its implementation of [J]technology innovation and.2015 (1)

2. Li Yao Yiran. [P].201410841242.32015.04.08 is an electronic commerce logistics system and its sorting method

3. Peng Zhao. Automatic sorting method and system for express parcel [P].201410 303596.22014.10.15

4. Wang Yun, Yang Jun et al. Multi-functional small sorting device-Express sorting [P].201310751923.62014.04.16

5. Zheng Xiongsheng, Wang Nanyong. A kind of express parcel sorting machine with a single device [P].201420555721.42015.01.07 